Die indirekte Steuerung von Arbeitsprozessen prägt auch unsere Vorstellung von der Arbeit selbst. Christoph Bartmann beschreibt in diesem Zusammenhang drei Leitmotive, die für die veränderte Wahrnehmung der Arbeit kennzeichnend sind. Zum einen sind wir stets darauf bedacht, unsere Arbeit und den damit verbundenen Höhepunkt unserer Leistung auf die Zukunft hin zu richten: »Den Gipfel unserer Leistungsfähigkeit oder Performance haben wir stets noch vor uns. " (Bartmann 2012: 219) Zum anderen haben wir das Bedürfnis, auf unsere Arbeit hinweisen $\mathrm{zu}$ müssen, denn »wir arbeiten nicht nur [...], wir zeigen oder weisen nach, während wir arbeiten, >dass` wir arbeiten, und sind schon deshalb niemals selbstvergessen.« (a.a.O.: 223 [Herv. i. O.]) Schließlich werden wir in der Arbeit aufgefordert, "'glatte<, visuell-grafische, problem- und widerspruchsfreie Formen der Präsentation« (a.a.O.: 239 [Herv. i. O.]) zu leisten, um den Anforderungen unserer Träger - im öffentlichen Dienst wie im Unternehmen - zu entsprechen. Dadurch entsteht eine Standardisierung von Arbeitsprozessen und Lebensgewohnheiten. Die omnipräsente und erforderliche Leistung (Performance), Hinweisung (Indikation) und Selbstdarstellung (Präsentation) haben das Office, den gegenwärtigen Lebensraum, in einen Ort verwandelt, in dem wir als Subjekte unsere Unterdrückung erleben und gleichzeitig unsere Befreiung realisieren (vgl. a.a.O.: 242f). Für diese neue Welt »der Präsentationen ohne Präsenz, der Performance ohne Ereignis, der Indikationen ohne Indiziertes, der großen, leeren Schau« (a.a.O.: 244) zahlen die Menschen einen hohen Preis. Gerade Burnout, die »Krankheit des positiven Denkens, der Optimierungen und der Performance« (a.a.O.: 246), soll das Resultat dieser Entwicklungen sein:

»Erst hat uns das positive Denken glauben gemacht, es gäbe für uns kein Limit, nun begegnen wir uns wieder als Performance-Junkies in der Kurklinik und üben uns, wie man jetzt immer häufiger hört, in >Achtsamkeit«. Die schädlichen Folgen positiven Denkens sollen offenbar durch noch mehr positives Denken behoben werden.«(a.a.O.: $265 f$ [Herv. i. O.])

\title{
3 Soziokultureller Blick auf das Burnout-Syndrom
}

Die gesellschaftstheoretische Kritik des Burnout-Syndroms und vergleichender psychischer Störungen, wie Boreout-Syndrom, Borderline, ADHS oder Depression, bezieht sich auf verschiedene Entwicklungen des modernen und postmodernen Zeitalters. Die soziologischen und kultursoziologischen Auslegungen fokussieren insbesondere auf die Verhältnisse, in die das Individuum eingebettet ist und die seine Subjektformen bestimmen. 


\subsection{Die Sorgen des Arbeitssubjekts}

Das Individuum als modernes Arbeitssubjekt unterliegt den Ansprüchen an die Identifikation mit der Arbeit. Diese Ansprüche werden in den gegenwärtigen Unternehmen durch eine omnipräsente Konkurrenz gestärkt, welche »die Langsameren/Schwächerern/Älteren latent unter den Erwartungsdruck der Schnelleren/Stärkeren/Jüngeren [setzt].« (Burisch 2014: 243) Daraus resultiert die Sorge eines jeden Arbeitssubjekts um die eigene Beschäftigungsfähigkeit (engl. employability), die ein ständiges Nachdenken über Risiken, Nebenwirkungen und Pläne B erfordert, sowie eine ständige Vernetzung, Flexibilität und Multifunktionalität voraussetzt (vgl. a.a.O.: 245). Die Sorge um sich führt dann nicht selten zu Identitätskrisen, die wiederum »auf veränderte gesellschaftliche Lebensbedingungen im globalisierten Netzwerkkapitalismus verweisen. Diese stellen Anforderungen an die alltägliche Identitätsarbeit dar, mit denen viele Menschen nicht mehr zu Recht kommen.« (Keupp 2010a: 45) Diese Anforderungen lassen sich an verschiedenen Erfahrungskomplexen, die für den Alltag prägend sind, verdeutlichen. $\mathrm{Zu}$ solchen gegenwärtigen Erfahrungen gehören z.B.

- eine Beschleunigung und Verdichtung des Alltags, die »zu den Grundgefühlen beitragen, getrieben $\mathrm{zu}$ sein, nichts auslassen zu dürfen, immer auf dem Sprung sein zu müssen, keine Zeit zu vergeuden und Umwege als Ressourcenvergeudung zu betrachten« (a.a.O.: 46; vgl. auch Rosa 2013);

- eine selbstunternehmerische Wahrnehmung meines Lebens, in der ich »für meine Gesundheit, für meine Fitness, für meine Passung in die Anforderungen der Wissensgesellschaft selbst zuständig [bin] « (Keupp 2010a: 46; vgl. auch Bröckling 2007);

- eine Deregulierung von Rollenschemata, die »einerseits als Gewinn an selbstbestimmter Lebensgestaltung verstanden wird, die aber andererseits in die Alltagswelten eine Unsicherheit hineinträgt, die nicht immer leicht akzeptiert und ertragen werden kann« (Keupp 2010a: 46);

- eine unabschließbare Arbeit an eigener Identität, die »die dazu erforderlichen Lebenskonzepte in einem sehr viel höheren Maße [braucht]« (a.a.O.: 47), als es für die Menschen früherer Generationen üblich war;

- eine Angst aus dem gesellschaftlichen Leben ausgegrenzt zu sein, welche viele Menschen bestimmt, weshalb diese »oft bereit sind, sich an Bedingungen anzupassen, die ihnen nicht gut tun« (ebd.);

- eine Suche nach einer Sicherheitsgesellschaft, die den Anspruch hat, »alles Unberechenbare, Uneindeutige, Ambivalente, Fremde und Störende zu beseitigen « (ebd.), um eine berechenbare und eindeutig geordnete Welt zu schaffen (vgl. Beck 1986; Münkler/Bohlender/Meurer 2010); 
- eine durch den kapitalistischen Wandel zur Monetarisierung, Ökonomisierung und >Vertriebswirtschaftlichung veränderte Arbeit- und Lebensqualität, die »nur noch in Geldwert ausgedrückt werden« scheint (Keupp 2010a: 48).

Auf der anderen Seite bestimmen die gesellschaftlichen Veränderungen die individuellen Ansprüche dahingehend, dass die Arbeitssubjekte diese als ihre eigenen entziffern. Dadurch erheben merkwürdigerweise wir selbst, als Arbeitssubjekte, einen Anspruch auf die Burnout-Krankheit, indem wir »an dieser neuen Welt des Change nicht nur teilnehmen, sondern teilnehmen swollen und Ziele haben, weil wir Prozesse optimieren wollen und überhaupt einer besseren Welt entgegentaumeln." (Bartmann 2012: 248f [Herv. i. O.]) Das BurnoutSyndrom zeichnet sich demnach durch seine positiven, erstrebenswerten und modernen Merkmale aus: »es ist eine Krankheit der neuen, storygetriebenen Bürokratie« (a.a.O.: 253), in der »nicht von Defiziten und Konflikten erzählt [wird], auch nicht von Unzulänglichkeiten und von Versagen [...], sondern von einer exzessiven Leistungsfähigkeit und -bereitschaft.« (Ebd.) Im Burnout überlappen sich die Pathologie und die Gesundheit zugleich, denn es ist der »bessere, der optimierte und also überbeanspruchte Mensch der idealtypische Burnout-Fall.« (a.a.O.: 259)

\subsection{Das Burnout-Syndrom als gesellschaftlicher Geisteszustand}

Nicht nur Pathologie und Gesundheit, sondern auch das Individuelle und das Gesellschaftliche verschmelzen im Burnout-Syndrom. Seelische Gesundheit hat in den letzten Jahrzehnten ihren sozialen Status verändert: „Sie gehört zu einer allgemeinen gesellschaftlichen Haltung, sie kennzeichnet eine bestimmte Atmosphäre unserer Gesellschaften, sie ist ein >Geisteszustand.« (Ehrenberg 2011: 21f [Herv. i. O.])

Im Geist der Französischen psychoanalytischen Schule betont Alan Ehrenberg, einer der bedeutendsten Soziologen, die sich mit der Problematik seelischer Störungen befassen, dass die gegenwärtigen Veränderungen und ihr Einfluss »auf den Aufbau des Subjekts und den Ausdruck des Leidens « (a.a.O.:321) auf das » Ende der Vertikalität, das Absterben der hierarchischen Gesellschaft, das vom Verfall der patriarchalischen Funktion bezeugt wird « zu verweisen sind (ebd. [Herv. i. O.]). Das Patriarchat hatte eine symbolische Funktion inne und begründete die gesellschaftliche Autorität. Die symbolische Stellung eines Vaters, einer Autorität »erfüllt seine $>$ normative Funktion nicht mehr. Auf diesem gesellschaftlichen Defizit gedeihen die neuen Pathologien.« (Ebd. [Herv. i. O.])

Das Ende der Vertikalität führte zum Ende der Unterwerfung unter die gesellschaftliche Pflicht. Dadurch verlor der Mensch »das Rückgrat, das ihn in der Gesellschaft verankerte und seine Begehrlichkeiten mäßigte. Der Verfall der Autorität der Gesellschaft lässt sich in der Ersetzung der alten Zwangshierarchien durch 
rein horizontales und gleichberechtigtes Schema der gesellschaftlichen und politischen Identifikation`(Schneider 2002: 183) erkennen« (a.a.O.: 325f). Und man kann sich nicht, so Ehrenberg, »von der Vertikalität befreien, ohne ganz konkret einen affektiven Preis dafür zu zahlen.«(a.a.O.: 322) Der Preis dafür sind die neuen Pathologien des Ideals, »jene Charakterneurosen, die die Unglücksfälle der Horizontalität sind, wie die Übertragungsneurosen die Pathologien der Vertikalität waren.« (a.a.O.: 322f) Wie er weiter in Bezug auf Michel Schneiders Big Mother (2002) betont, folgten auf »die >Krankheiten des Vaters (Zwangsneurose, Hysterie, Paranoia) [...] im großen Maß die >Krankheiten der Mutter (Grenzzustände, Schizophrenien, Depressionen).«(Schneider 2002: 112 zit.n. Ehrenberg 2011: 323) Diese Pathologien »betreffen das Ideal in dem Sinne, dass sie durch einen >wirklichen Verfall der väterlichen Imago in der Gesellschaft verursacht werden und somit durch den Verfall von Institution, Symbol, Transzendenz, Hierarchie, Grenze, die eine Begriffsfamilie bilden.« (Ehrenberg 2011: 323 [Herv. i. O.]) ${ }^{10}$

Des Weiteren hat die Entwicklung von Charakterneurosen (Krankheiten der Mutter), welche das vorherige Interesse an Übertragungsneurosen (Krankheiten des Vaters) ersetzt hat, neue Formen des Ausdrucks von Gefühlen mit sich gebracht und die Transformation der individuellen Neurosen in gesellschaftliche Probleme bewirkt (vgl. a.a.O.: 498). ${ }^{11}$ Und diese Art von Problemen charakterisiert unser Zeitalter, das, pathologisch gesehen, "weder bakteriell noch viral, sondern neuronal bestimmt « ist (Han 2010: 5). Das heißt, dass die neuronalen Erkrankungen wie Depression, Burnout oder ADHS »keine Infektionen [sind], sondern Infarkte, die nicht durch die >Negativität $<$ des immunologisch Anderen, sondern durch ein Übermaß an >Positivität< bedingt sind.« (ebd. [Herv. i. O.])

Anstelle der durch die Negativität bestimmten Gesellschaftskonstellation der Andersheit und Fremdheit, tritt nämlich das Paradigma der durch das Übermaß an Positivität erzeugten Differenz. Diese ruft keine Immunreaktion hervor. »Die postimmunologische, ja postmoderne Differenz macht nicht mehr krank. Auf der immunologischen Ebene ist sie das >Gleicheく. Der Differenz fehlt gleichsam der

10 Die neuen Pathologien stellen außerdem den kulturellen Code der Gesellschaft dar. Denn »seit der Aufklärung hat sich der soziale Status des psychischen Leidens dahingehend gewandelt, dass die vorherrschenden Krankheitsbilder nicht nur individuelles Leiden, sondern auch die Dilemmata des Soziallebens zum Ausdruck bringen. Losgelöst vom Krankenbett etablierte sich eine Tradition der psychopathologischen Kulturkritik, in der Krankheitsbilder-Degeneration, Schizophrenie oder Narzissmus - als kulturelle Codes figurierten.«(Bernet 2012: 195)

Diese Transformation wird heutzutage am deutlichsten am Fall der Depression sichtbar: »Über die Depression wird regiert. Denn die Weisen der veränderten Selbstproblematisierung korrespondieren mit Steuerungsmechanismen, in denen Depressionsbekämpfung zum snationalen Cesundheitsziel wird und Initiativen zur Beförderung der psychischen Cesundheit der Bevölkerung staatlich initiiert werden.« (Abels 2015: 280 [Herv. i. O.]) 
Stachel der Fremdheit, der eine heftige Immunreaktion auslösen würde.« (a.a.O.: 7 [Herv. i. O.]) Richtete sich die immunologische Abwehr des Organismus »immer gegen das Andere oder das Fremde im emphatischen Sinne« (a.a.O.: 11), so sind Erschöpfung und Ermüdung »Erscheinungen einer >neuronalen Gewalt<, die insofern nicht viral ist, als sie auf keine immunologische Negativität zurückzuführen ist« (a.a.O.: 12 [Herv. i. O.]), sondern eher auf das »Zuviel am Gleichen , [an das] Übermaß an Positivität.« (ebd. [Herv. i. O.])

In Kritik an Foucaults Disziplinargesellschaft zeigt der Kulturphilosoph $\mathrm{Bu}$ yung Chul Han, dass das heutige Leistungssubjekt »Herr und Souverän seiner Selbst ist.«(a.a.O.: 22) Jedoch führt dieser Wegfall von der Herrschaftsinstanz nicht $\mathrm{zu}$ mehr Freiheit für das Individuum. Vielmehr lässt er »Freiheit und Zwang zusammenfallen. So überlässt sich das Leistungssubjekt der >zwingenden Freiheit oder dem >freien Zwang zur Maximierung der Leistung.« (ebd. [Herv. i. O.]) Wobei die Freiheit im eigentlichen Sinne »an die Negativität gebunden ist. Sie ist immer eine Freiheit vom Zwang, der vom immunologisch Anderen ausgeht. Wo die Negativität dem Übermaß an Positivität weicht, verschwindet auch die Emphase der Freiheit, die dialektisch der Negation der Negation entspringt.« (Ebd.) Aus dieser paradoxen Freiheit ließen sich dann die psychischen Krankheiten als pathologische Manifestationen ausdeuten (vgl. a.a.O.:23). Es ist das Zusammenfallen der Freiheit und des Zwangs, des Herrn und des Knechts, des Gefangenen und des Aufsehers seines Selbst, die zu einer Art freiwilliger Ausbeutung führt (vgl. a.a.O.:35f), welche »auch ohne Herrschaft möglich [ist].«(a.a.O.: 36)

\subsection{Das Burnout-Syndrom - eine Modeerscheinung?}

Inwiefern es sich beim Burnout-Syndrom tatsächlich um eine Krankheit handelt und was darunter im Licht seiner medialen Darstellung (vgl. Hillebrand 2013) und seinen historischen Parallelen (vgl. Becker 2017) eigentlich zu verstehen ist, fragt sich Frieder Vogelmann und behauptet vier Nichtexistenzweisen dieser psychischen Störung: »Burnout ist keine >individuelle Krankheit<, weil es (1) keine >Krankheit beziehungsweise (2) keine sindividuelle Krankheit ist, weil Burnout (3) verschiedene sandere Krankheiten bezeichnet oder weil Burnout (4) >mehr< als eine Krankheit ist.«(Vogelmann 2013: 149 [Herv. i. O.])

- Der ersten Nichtexistenzbehauptung liegt einerseits die Tatsache zugrunde, dass Burnout bis zum heutigen Tag offiziell nicht als eine Krankheit deklariert wurde; so in den internationalen auch als in nationalen Klassifikationen. ${ }^{12}$ An-

12 Nach der in 2019 stattgefundenen Neufassung der Internationalen Klassifikation der Krankheiten (ICD) wird allerdings das Burnout demnächst (ab 2022) als Krankheit eingestuft, die durch drei Dimensionen gekennzeichnet wird, und zwar durch das Gefühl von Erschöpfung, 
dererseits handelt es sich nicht um eine Krankheit, weil es »nicht als solche klassifiziert >ist<, sondern weil sie nicht als solche klassifiziert werden `sollte «." (ebd. [Herv. i. O.]) Als Grund nennt Vogelmann die Beobachtung, laut welcher »ein Mensch, der an seiner Arbeit ausbrennt, [...] in unserer Gesellschaft vielleicht ein kostbares und sensibles Barometer der kaputten gesellschaftlichen Bedingungen und der damit korrelierten kaputten Arbeitsverhältnisse [ist] der einzige, der gesund reagiert auf kranke Verhältnisse: leidend.« (Rösing 2003: 239 zit.n. Vogelmann 2013: 150)

- Was die zweite Behauptung angeht, so stellt er fest, dass Burnout »keine >individuelle<, sondern >soziale Krankheit ist." (Vogelmann 2013: 150 [Herv. i. O.]) Ausgehend von der Marx'schen Theorie, in welcher Burnout »die Vergegenständlichung der Produktionsmittel des Sozialarbeiters [ist] (Karger 1981: 275 zit.n. Vogelmann 2013: 151), wo die Fähigkeiten des Arbeiters »Zwischenmenschliche Beziehungen einzugehen, [...] zu einer handelbaren Ware [werden] (ebd.) und wo die Distanzierung des Sozialarbeiters von seinem Klienten zu einer Verdinglichung führt, »die den Arbeiter von der authentischen Ausübung seiner Fähigkeiten entfremdet (ebd.), bezeichnet er das Burnout als eine »Entfremdung und daher keine individuelle Krankheit, sondern eine soziale Pathologie.«(Vogelmann 2013: 151)

- Die dritte Nichtexistenzbehauptung legt nahe, dass Burnout wegen seiner mangelnden Abgrenzung meigentlich verschiedene sandere, nicht unter einen einzigen Oberbegriff zu fassende Krankheiten bezeichnet.« (ebd. [Herv. i. O.]) Eine differentialdiagnostische Beurteilung zeigt nämlich, dass »Burnout als Phänomen >nebulös und vielfältig` wirkt, weil >unscharf definiert [ist], was Burnout eigentlich ist - und differentialdiagnostisch bedeutsam: was Burnout nicht ist «« (Korczak/Kister/Huber 2012: 99 zit.n. Vogelmann 2013: 151)

- Letztens ist Burnout mehr als Krankheit, eine sog. sprechende Krankheit. Es stellt ein »sprachliches Vehikel [dar], das geeignet ist, persönliches Leiden des Individuums, vornehmlich in und an der Arbeitswelt, zum Ausdruck zu bringen.« (Hillert \& Marwitz 2006: 282 zit.n. Vogelmann 2013: 151)

Demgegenüber hält Ulrich Bröckling das Burnout-Syndrom für eine Krankheit unserer Zeit, die neben anderen Zivilisationskrankheiten zu verorten ist. Solche Krankheiten zeichnen sich für ihn »nicht nur durch gehäuftes Auftreten, sondern vor allem dadurch aus, dass ihre Symptome den Zeitgenossen einen Spiegel vorhalten. Sie reflektieren, was diese fürchten, worunter sie leiden und woran sie scheitern - und zeigen dadurch zugleich rex negativo die Fluchtpunkte gegenwärtiger

durch eine zunehmende geistige Distanzierung bzw. negative Haltung gegenüber des eigenen Jobs gleichwie durch ein verringertes berufliches Leistungsvermögen (vgl. Dear Employee 2019). 
Lebensführung, die Vorstellungen darüber, wie die Einzelnen sich heute selbst begreifen, wie sie an sich arbeiten und für sich sorgen sollen.« (Bröckling 2013: 181 [Herv. i. O.]) Mit Blick auf die Sinnbilder, welche mit dem Burnout-Syndrom evoziert werden, lassen sich seine Konturen in drei Bildern konkreter aufzeichnen:

- Das Ausgebranntsein erinnert zuerst an das Bild des Feuers, welches aufgrund seines Nutzens aber auch seiner Gefahr ambivalenter Natur ist (vgl. a.a.O.: 182). Der Brennprozess mit seiner entzündeten Flamme und der am Ende übrig gebliebenen Asche ermöglicht der Feuer-Metapher "gleichermaßen auf die vergangene Verausgabung wie auf die Unfähigkeit, sie fortzusetzen« (ebd.) zu verweisen.

- Eine weitere Metapher, »eng verwandt mit dem Bild der individuellen Leistungs- beziehungsweise Lebensflamme, die brennt oder eben erloschen ist, ist der Topos des Burnouts als einer >persönlichen Energiekriseく, die auf einer Dysbalance zwischen Kraftzufuhr und -verbrauch beruht.« (a.a.O.: 185 [Herv. i. O.]) Diese Metapher einer Menschenmaschine »impliziert die Vorstellung eines Subjekts, das sich aufspaltet, einerseits in eine technische Apparatur, andererseits in eine Instanz, die diese beobachtet und sie entweder $\mathrm{zu}$ optimieren sucht oder ihren Rückbau zu einem fühlenden Wesen einleitet.« (a.a.O.: 188)

- Schließlich verweist die Metapher des Hamsterrads (vgl. a.a.O.: 189) auf das asymmetrische Gleichgewicht zwischen Stress und Entspannung. In dem Diskurs um Burnout wird einerseits »ein zivilisationskritisch-pessimistisches Bild der Gegenwart gezeichnet, wie es düsterer kaum ausfallen könnte. Weil das aber kaum zu ertragen wäre und in Fatalismus münden müsste, wechselt man andererseits das Register, sobald es um präventive und therapeutische Maßnahmen geht, und propagiert einen pragmatischen Aktivismus. Der changiert zwischen verhältnis- und verhaltensbezogenen Konzepten und landet am Ende - wen wundert's? - bei der Forderung nach einem integrativen Ansatz, der sowohl die Belastungen reduzieren wie die Belastbarkeit steigern und gesellschaftliche Anforderungen und persönliche Ressourcen wieder ins Gleichgewicht bringen soll.« (a.a.O.: 193) Und ein Gleichgewicht herrscht erst dann, »wenn gegenstrebige Kräfte - in diesem Fall: Anspannung und Entspannung, Motivationssystem und Stresssystem, Arbeitsansprüche und Ressourcen, Leistung und Wertschätzung - einander neutralisieren.«(a.a.O.: 195f)

Alle drei Bilder umschreiben das Burnout-Syndrom in seiner gesellschaftlichen Wahrnehmung. Ob das Burnout-Syndrom als eine Krankheit bzw. Störung empfunden wird oder nicht, ändert nichts an seinen Auswirkungen, die sowohl auf individueller wie auf gesellschaftlicher Ebene zu erheblichen Schäden führen. 